\title{
The measurement of overhead conductor's sag with DLT method
}

\author{
Fang $\mathrm{Ye}^{1 \dagger}$, Meng Tian ${ }^{2}$, Xing Zhang ${ }^{2}$, \\ Zhi-Yong $\mathrm{Gan}^{3}$, Xin $\mathrm{He}^{3}$ and Zhang-Qi Wang ${ }^{2}$ \\ ${ }^{1}$ Electric Power Research Institute, State Grid Tianjin \\ Electric Power Company, Tianjin, 300384, China \\ E-mail:18630956321@163.com \\ ${ }^{2}$ Department of Mechanical Engineering, North China \\ Electric Power University, Baoding, 071003, China \\ ${ }^{3}$ Electric power science and Technology Development Co. \\ Ltd. of Tianjin city, Tianjin 300384, China
}

\begin{abstract}
The overhead conductor sag calculation is put forward combined with image analysis technology based on DLT theory in this paper. Moreover, the simulation experiment is carried on and the measurement error is also analyzed. The attentions needed in practical operation are also provided. The result shows that the method provided in this paper is a quick, efficient and practical measurement method for transmission line inspection.

Keywords: Direct Linear Transformation; Overhead Conductor; Sag.
\end{abstract}

\section{Introduction}

The electric power system is the foundation of China's economic construction, and it is also an important guarantee for the national life. Sag is one of the important parameters for operation and maintenance of transmission lines, so it is necessary to control the sag of overhead conductor within the allowable range. If the sag is too small, the wire stress will be increased, which will cause more vibration and the break of transmission line, and even the damage of electric power tower. If the sag is too large, the safe distance between the wire and the ground is not enough, and the discharge phenomenon will be taken place, and even the phenomenon of short twist is occurred to conductor galloping. And furthermore, sag is one of the limiting factors to increase the transmission capacity of overhead transmission lines. The transmission capacity can be adjusted by the real-time dynamic measurement of the sag, which could release the conservative transmission capacity to achieve the purpose of dynamic capacity increase [1]. Therefore, it is of great significance to measure the sag of overhead transmission lines regularly to ensure the safe operation of the whole power grid.

The traditional methods of sag measurement include angle method, equal 
length method and relaxation plate observation method, etc, but these methods are very inconvenient to operate, and have large human error and low measurement efficiency. In reference [2], the local meteorology parameters are considered as the control condition, and the wire sag is calculated by measuring the stress or inclination of conductor in combination with the conductor state equation, but this method requires a large investment and has high cost. Chris Menasha Bonus in [3] studies DGPS technology for conductor sag measurement. This method uses four satellites to locate the position of Rover that can be used in any environment hanging fixed point for overhead conductor sag measurement, however, the price of this measurement method is very expensive, and the power consumption is also high. The reference [4] shows that the spacer is used as a template to match the spacer of the conductor through image processing, then three-dimensional coordinate transformation is carried out for the match point, the conductor sag is calculated by curve fitting of world coordinates of the match point. As the spacer could be out of shape in different positions and is easily affected by the environment during the matching procedure, it is also difficult to measure transmission lines sag in practical application.

In this paper, a new method is put forward to measure the overhead conductor sag based on two-dimensional plane image analysis. In the present method, a marker on the measuring spot is used and the coordinates of the marked point is obtained by a photograph analysis method.

\section{Director Linear Transformation}

\subsection{Camera imaging model}

The camera imaging model is an important part of the measurement system. In the monocular vision, according to requirements of the measurement and the actual situation, the camera imaging model can be divided into vertical projection model, weak perspective imaging model, pinhole imaging model, and so on. Among of them, the pinhole imaging model is the closest to the actual situation [5-7]. So this paper selects the pinhole model as camera imaging model.

The principle of pinhole imaging model is shown in the Fig.1. Spatial point $\mathrm{P}$ passes through the optical center $\mathrm{O}$, and $\mathrm{PO}$ intersect at $\mathrm{p}$ point with in the projection plane.

The camera coordinate of spatial point $\mathrm{P}$ is denoted as $\left(X_{C}, Y_{C}, Z_{C}\right)$, the space coordinate of space coordinates system $O_{w}-X_{W} Y_{w} Z_{w}$ is $P(X, Y, Z)$, corresponding to the image pixel coordinate of the projection point $p(u, v)$. 


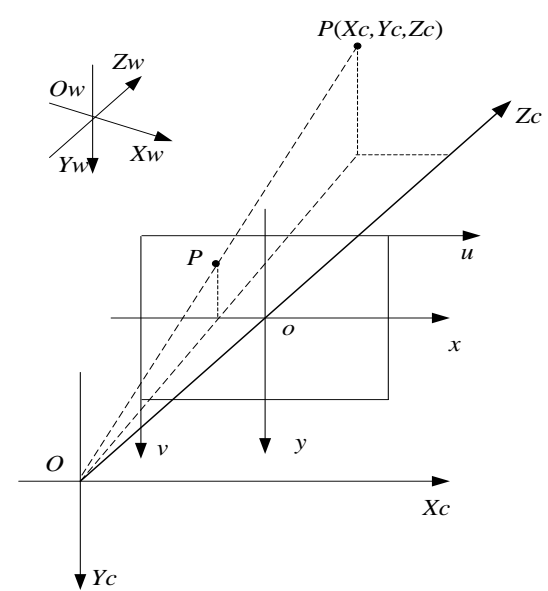

Fig. 1. This is the principle of pinhole imaging model.

\subsection{DLT mathematical model}

Direct linear transformation is obtained by the collinear equation of image point, optical center and spatial point. The transformation relationship between image coordinates and spatial coordinates is established through DLT. During the calculation, the internal and external parameters of the camera are not required, and the features of the DLT are simple and stable.

Thus, the following relationship can be obtained according to DLT theory:

$$
\left\{\begin{array}{l}
u=\frac{L_{1} X+L_{2} Y+L_{3} Z+L_{4}}{L_{9} X+L_{10} Y+L_{11} Z+1} \\
v=\frac{L_{5} X+L_{6} Y+L_{7} Z+L_{8}}{L_{9} X+L_{10} Y+L_{11} Z+1}
\end{array}\right.
$$

Here, the space coordinate of object points is $(X, Y, Z)$, the coordinate of image pixel coordinate system is $(u, v)$. And $L_{i}$ is the element of the transformation matrix $\boldsymbol{L}$.

It is very easy to derive the two-dimensional DLT method from the threedimensional DLT solution, that is, $Z$ is appointed as zero, the two-dimensional DLT mathematical model can be obtained from,

$$
\left\{\begin{array}{l}
u=\frac{L_{1} X+L_{2} Y+L_{4}}{L_{9} X+L_{10} Y+1} \\
v=\frac{L_{5} X+L_{6} Y+L_{8}}{L_{9} X+L_{10} Y+1}
\end{array}\right.
$$


Note that there are only 8 variables in the two-dimensional DLT mathematical model, while the 3D DLT mathematical model has 11 variables, so the twodimensional DLT has the advantages of small amount of calibration points, simple operation and less computation. From the two-dimensional DLT model, if the coordinates of 4 points in pace scene and their corresponding pixel coordinates are available, 8 equations can be obtained and the transformation matrix $\boldsymbol{L}$ can be known by solving these 8 equations.

\section{Object Spatial Coordinates Calculation}

In the calculation of the coordinates of spatial point, the transform matrix $\boldsymbol{L}$ should be calculated first, then the object coordinates can be obtained by $\boldsymbol{L}$, finally, the conductor sag can be obtained by fitting the object coordinates.

\subsection{Resolution of transformation matrix}

When the spatial coordinates of $n$ landmarks and corresponding pixel coordinates are available, $2 \times n$ equations can be obtained as in following,

$$
\left[\begin{array}{cccccccc}
X_{1} & Y_{1} & 1 & 0 & 0 & 0 & -u_{1} X_{1} & -u_{1} Y_{1} \\
0 & 0 & 0 & X_{1} & Y_{1} & 1 & -X_{1} v_{1} & -v_{1} Y_{1} \\
& & & & \cdots & \cdots & & \\
X_{n} & Y_{n} & 1 & 0 & 0 & 0 & -u_{n} X_{n} & -u_{n} Y_{n} \\
0 & 0 & 0 & X_{n} & Y_{n} & 1 & -X_{n} v_{n} & -v_{n} Y_{n}
\end{array}\right] \boldsymbol{L}=\left[\begin{array}{c}
u_{1} \\
v_{1} \\
\vdots \\
u_{n} \\
v_{n}
\end{array}\right]
$$

where

$$
\boldsymbol{L}=\left[\begin{array}{llllllll}
L_{1} & L_{2} & L_{4} & L_{5} & L_{6} & L_{8} & L_{9} & L_{10}
\end{array}\right]^{T}
$$

Then, the transform matrix is

$$
\boldsymbol{L}=\left(\boldsymbol{A}^{T} \boldsymbol{A}\right)^{-1} \boldsymbol{A}^{T}\left[\begin{array}{c}
u_{1} \\
v_{1} \\
\vdots \\
u_{n} \\
v_{n}
\end{array}\right], \boldsymbol{A}=\left[\begin{array}{cccccccc}
X_{1} & Y_{1} & 1 & 0 & 0 & 0 & -u_{1} X_{1} & -u_{1} Y_{1} \\
0 & 0 & 0 & X_{1} & Y_{1} & 1 & -X_{1} v_{1} & -v_{1} Y_{1} \\
& & & & \cdots & \cdots & & \\
X_{n} & Y_{n} & 1 & 0 & 0 & 0 & -u_{n} X_{n} & -u_{n} Y_{n} \\
0 & 0 & 0 & X_{n} & Y_{n} & 1 & -X_{n} v_{n} & -v_{n} Y_{n}
\end{array}\right]
$$

In order to calculate the 8 variables of the transformation matrix $\boldsymbol{L}$, the spatial coordinates of at least 4 marker points and the corresponding pixel coordinates should be known.

\subsection{Calculation of object point coordinate}

When the transformation matrix and the spatial coordinates of $n$ landmarks and the corresponding pixel coordinates are known, the spatial coordinates of the 
object can be calculated according to,

$$
\left[\begin{array}{c}
X_{1} \\
Y_{1} \\
\vdots \\
X_{n} \\
Y_{n}
\end{array}\right]=\left(\boldsymbol{B}^{T} \boldsymbol{B}\right)^{-1} \boldsymbol{B}^{T}\left[\begin{array}{c}
u_{1}-L_{4} \\
v_{1}-L_{8} \\
\vdots \\
u_{n}-L_{4} \\
v_{n}-L_{8}
\end{array}\right], \boldsymbol{B}=\left[\begin{array}{cc}
L_{1}-u_{1} L_{9} & L_{2}-u_{1} L_{10} \\
L_{5}-v_{1} L_{9} & L_{6}-v_{1} L_{10} \\
\cdots & \cdots \\
L_{1}-u_{n} L_{9} & L_{2}-u_{n} L_{10} \\
L_{5}-v_{n} L_{9} & L_{6}-v_{n} L_{10}
\end{array}\right]
$$

\subsection{Overhead line curve fitting}

The two-dimensional spatial coordinates of each point of the overhead conductor can be obtained by the Eq. (5), and then the calculation model of the conductor can be calculated by the method of data regression.

The calculation model of overhead conductor can be described as catenary or parabolic equation [8]. In order to avoid the hyperbolic function and simplify the calculation, this paper adopts parabolic equation. The quadratic parabolic equation model of overhead conductor is shown as

$$
y=k_{0}+k_{1} x+k_{2} x^{2}
$$

Here, $(x, y)$ is the spatial coordinate of a point on the overhead line, and $k_{0}$, $k_{1}, k_{2}$ are undetermined coefficients. Using the nonlinear regression algorithm of least square to fit the feature points of the conductor, the value of the undetermined coefficients can be calculated, and then the sag of overhead conductor also can be obtained easily.

\section{Simulation Experiment Research}

In order to verify the accuracy and feasibility of the overhead line sag calculation method, the simulation experiment is carried on. For convenience, the height between the ground and the conductor near stadia rod is taken as the measuring object. In addition, the influence of distribution of the identification points on the measurement accuracy has also been analyzed.

Since the smooth cable, with the characteristics of uniform load, inextensibility and fully flexibility, is accord with the state of overhead line, it is used to carry on the experiment instead of overhead line. The experimental image is shown in Fig.2. The simulation conductor of this experiment is a constant height suspension, and the related parameters are measured by the stadia rod. The ground clearance of conductor is $200 \mathrm{~cm}$, and the side length value of checkerboard is $5 \mathrm{~cm}$ square. 


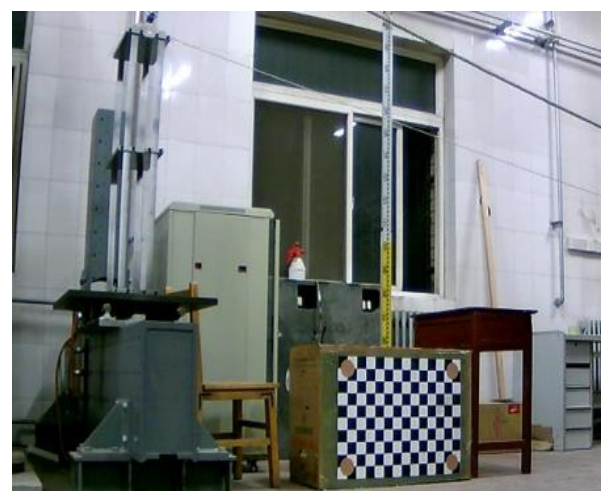

Fig. 2. This is the simulation of experimental based on DLT.

If the checkerboard is placed in the conductor plane, the spatial coordinate of each point of the conductor can be calculated according to the DLT theory. The flowchart of the procedure is presented in Fig. 3.

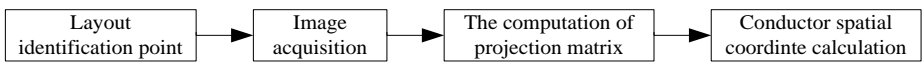

Fig. 3.This is the flow chart of the experimental procedure.

First, the calibration points on the checkerboard is chosen and the transformation matrix $\boldsymbol{L}$ is calculated according to the Eq. (4). Then, the measurement point in the conductor is selected, and the two-dimensional spatial coordinates is calculated according to the Eq. (5). In the experiment, the calibration points of different distribution forms are selected and divided into six groups, that is, $A 1$ $A 4, B 1-B 4, C 1-C 4, D 1-D 4, E 1-E 4, F 1-F 4$, respectively corresponding $1 \times 1,2 \times 2$, $4 \times 4,6 \times 6,8 \times 8,10 \times 10$ square. The distribution of calibration points are shown in Fig. 4:

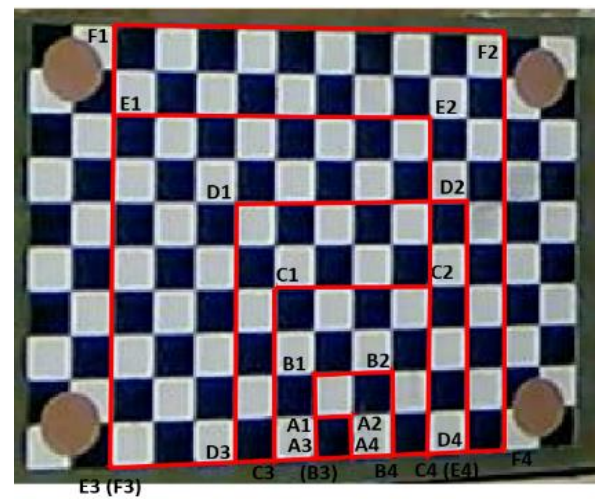

Fig. 4. This is the distribution of calibration points on the checkerboard

The actual value of the distance between conductor and ground is $200 \mathrm{~cm}$, while the calculation results of the identification points with different distribution 
conditions are shown in table 1.

Table 1. This is the experimental result and the measurement error.

\begin{tabular}{l|l|l|l|l|l|l}
\hline Size of calibration grid & $1 \times 1$ & $2 \times 2$ & $4 \times 4$ & $6 \times 6$ & $8 \times 8$ & $10 \times 10$ \\
\hline Measured value $(\mathrm{cm})$ & 141.23 & 166.50 & 185.88 & 186.87 & 196.51 & 197.82 \\
\hline Relative error $(\delta)$ & $29.38 \%$ & $16.75 \%$ & $7.07 \%$ & $6.57 \%$ & $1.60 \%$ & $1.09 \%$ \\
\hline
\end{tabular}

From the above results, it shows that the larger the surrounded area of calibration points in the image proportion is, the smaller the measurement error is. Moreover, the measured values are less than the actual value, which means there are some system errors.

\section{Conclusions}

A measurement method for overhead conductor sag is put forward using twodimensional image measurement based on the DLT theory. The influence of the distribution of the calibration points on the measuring accuracy is analyzed, which shows the measurement accuracy is higher when the identification point is larger than $1 / 4$ in the image. The proposed method can calculate the conductor sag with the characteristics of simple operation, good accuracy, high efficiency and practicability, which avoids the disadvantages of the traditional manual measurement method.

\section{References}

1. Forbes, Blake, D. Bradshow, and F. Campbell, Finding hidden capacity in transmission lines. Transmission \& Distribution World, 2002, 54, (9).

2. Wei Kong, Xiao-Guang Dai, Zhen-Wei Yang, Matlab realization of stress sag curve of overhead line. Electric Power, 2009, 42 (7): 46-49.

3. Mensah-Bonsu C, Krekeler U F, Heydt G T, etc, Application of the global positioning system to the measurement of overhead power transmission conductor sag. IEEE Transactions on Power Delivery, 2002, 17(1):273-278.

4. Wei-Guo Tong, Bao-Shu Li, Jin-Sha Yuan, etc. Method of transmission line sag measurement based on aerial image sequence. Proceedings of the CSEE, 2011, 16:115-120.

5. D.A.Forsyth, J.Ponce. Forsyth. Computer Vision: A Modern Approach. Prentice Hall Professional Technical Reference, 2002.

6. R. Szeliski, Richard. Computer Vision: Algorithms and Applications. Journal of Polymer Science Polymer Chemistry Edition 21.8 (2011):2601-2605.

7. M.S.Nixon, A.S.Aguado. Feature extraction and image processing. Beijing: Electronic Industry Publishing House, 2011:288-308.

8. Dian-Sheng Zhang. Design Manual of High Voltage Transmission Line in Power Engineering. Beijing: China Power Press, 2003. 